\title{
Author Correction: The SEIRS model for infectious disease dynamics
}

Ottar N. Bjørnstad, Katriona Shea, Martin Krzywinski and Naomi Altman

Correction to: Nature Methods https://doi.org/10.1038/s41592-020-0856-2, published online 4 June 2020.

In the version of this article initially published, the sum $\sigma+\mu+\alpha$ appeared in denominators in the expressions for $R_{0}$ and infectious period in the sentence immediately after the displayed equations. The correct sum in both cases is $\gamma+\mu+\alpha$. The error has been corrected in the PDF and HTML versions of the article.

Published online: 1 February 2021

https://doi.org/10.1038/s41592-021-01079-6

๑ Springer Nature America, Inc. 2021 